\title{
Post-Discharge Services for Different Diagnoses Than Index Hospitalization Predict Decreased 30-Day Readmissions Among Medicare Beneficiaries
}

\author{
Hyo Jung Tak, $P h D^{7}$, Li-Wu Chen, $P h D^{7}$, Fernando A. Wilson, $P h D^{7}$, \\ Andrew M. Goldsweig, MD², Dmitry Oleynikov, MD³, Michael Hawking, MD 4 , and \\ Ya-Chen Tina Shih, $P h D^{5}$
}

\begin{abstract}
'Department of Health Services Research and Administration, University of Nebraska Medical Center, Omaha, NE, USA; ${ }^{2}$ Division of Cardiovascular Medicine, University of Nebraska Medical Center, Omaha, NE, USA; ${ }^{3}$ Department of Surgery, University of Nebraska Medical Center, Omaha, NE, USA; ${ }^{4}$ Department of Internal Medicine, University of Chicago, Chicago, IL, USA; ${ }^{5}$ Department of Health Services Research, The University of Texas MD Anderson Cancer Center, Houston, TX, USA.
\end{abstract}

BACKGROUND: Efforts to reduce hospital readmissions include post-discharge interventions related to the illness treated during the index hospitalization (IH). These efforts may be inadequate because readmissions are precipitated by a wide range of health conditions unrelated to the primary diagnosis of the IH.

OBJECTIVE: To investigate the relationship between post-discharge health services utilization for the same or a different diagnosis than the IH and unplanned 30-day readmission.

DESIGN AND PARTICIPANTS: The study sample included 583, 199 all-cause IHs among 2014 Medicare fee-forservice beneficiaries. For all-cause IH, as well as individually for heart failure, myocardial infarction, and pneumonia $\mathrm{IH}$, we used multivariable logistic regressions to investigate the association between post-discharge services utilization and readmission.

MAIN MEASURES: The outcome was unplanned 30-day readmission. Primary independent variables were postdischarge services utilization, including institutional outpatient, office-based primary care, office-based specialist, office-based non-physician practitioner, emergency department, home health care, and skilled nursing facility providers.

KEY RESULTS: Among all-cause IH, 11.7\% resulted in unplanned 30 -day readmissions, and only $18.1 \%$ of readmissions occurred for the same primary diagnosis as IH. A substantial majority of post-discharge health services were utilized for a primary diagnosis differing from IH. Compared with no visit, institutional outpatient visits for the same primary diagnosis as IH (odds ratio [OR], 0.33; 95\% confidence interval [CI], 0.31-0.34) and for a different primary diagnosis than IH (OR, 0.36; 95\% CI, 0.35-0.37) were similarly strongly associated with decreased unplanned 30-day readmission. Primary care physician, specialist, non-physician practitioner, and home health care showed similar patterns. IH for heart

Prior Presentations None.

Received August 10, 2018

Revised November 5, 2018

Accepted May 1, 2019

Published online June 21, 2019 failure, myocardial infarction, and pneumonia manifested similar patterns to all-cause IH both in terms of postdischarge services utilization and in terms of its impact on readmission.

CONCLUSIONS: To reduce unplanned 30-day readmission more effectively, discharge planning should include post-discharge services to address health conditions beyond the primary cause of the IH.

KEY WORDS: all-cause index hospitalization; 30-day readmission; postdischarge services utilization; service diagnosis; Hospital Readmission Reduction Program.

J Gen Intern Med 34(9):1766-74

DOI: $10.1007 / \mathrm{s} 11606-019-05115-2$

(c) Society of General Internal Medicine 2019

\section{INTRODUCTION}

Unplanned 30-day hospital readmission is often considered a marker of poor quality care during an index hospitalization (IH) and constitutes a substantial proportion of potentially avoidable health care expenditures. ${ }^{1-7}$ Medical practitioners and policymakers have therefore implemented various interventions to improve the quality of inpatient care ${ }^{8-10}$ and to reduce readmissions. ${ }^{3-6,11-14}$

However, unplanned 30-day readmissions are not always due to suboptimal $\mathrm{IH}$ care: recently discharged patients are frequently readmitted for a wide range of health conditions unrelated to the $\mathrm{IH}^{7}{ }^{15-20}$ Therefore, current post-discharge interventions primarily focusing on the acute illness treated during IH may be insufficient. ${ }^{15,16}$ Indeed, literature suggests that, among patients whose primary diagnosis during $\mathrm{IH}$ placed them in the Centers for Medicare and Medicaid Services' (CMS) initial Hospital Readmission Reduction Program (HRRP), only $10.0-35.2 \%$ of readmissions were attributable to the same conditions as $\mathrm{IH}^{17}$ While this CMS program targets selected $\mathrm{IH}$ diagnoses, readmission may occur for any cause, whether related to the IH diagnosis or not. ${ }^{7,15-17}$

An understanding of patients' post-discharge health services utilization patterns and their impact on readmission is 
crucial to designing post-discharge interventions that are more effective at reducing readmission. ${ }^{21}$ Indeed, discharged patients may access various post-discharge services for both the IH diagnosis and other diagnoses. To our knowledge, however, no prior study has systematically investigated the relationship between post-discharge services utilization and readmission incorporating the relatedness of diagnoses between IH and post-discharge services.

This study used Medicare claims data for all-cause $\mathrm{IH}$ followed by all-cause unplanned 30-day readmission to examine the association between post-discharge services utilization and readmissions. In particular, we documented the extent to which post-discharge services were utilized for the same or a different diagnosis than $\mathrm{IH}$ and whether the impact of postdischarge services varied in terms of readmission prevention if services were provided for the same or a different diagnosis than IH.

\section{METHODS}

\section{Data Sources and Study Population}

Our study used 2014 Medicare claims including data from the Master Beneficiary Summary File, Medicare Provider Analysis and Review (inpatient and skilled nursing facilities), institutional outpatient (e.g., hospital outpatient departments, outpatient rehabilitation facilities, Federally Qualified Health Centers), carrier (emergency department [ED] and officebased health services across various medical provider specialties), and home health care. The initial study sample included Medicare fee-for-service (FFS) patients hospitalized for acute illness between February 1 and November 30. Hospitalizations that occurred in January and December were used to account for health services 30 days prior to $\mathrm{IH}$ and 30 days after discharge.

We defined all-cause IH using criteria from the CMS 2014 Measure Updates and Specification Report (CMS-MUSR). ${ }^{22,}$

${ }^{23}$ These criteria excluded hospitalizations from classification as an IH if (i) the primary diagnosis at discharge was medical treatment of cancer, rehabilitation, or psychiatric in nature; (ii) the discharge status was dead in hospital, left hospital against medical advice, or transferred to another acute care facility; and (iii) patients unenrolled in Medicare FFS plans or died within 30 days of discharge. The unit of analysis was $\mathrm{IH}$, and only hospitalizations subsequent to the IH were used to identify readmissions. When a patient was readmitted multiple times within 30 days of discharge from $\mathrm{IH}$, only the first readmission was counted and additional readmissions within this period were not counted as either new IH or readmission per CMS criteria. ${ }^{17,22}$ An individual could have multiple IHs if subsequent hospitalizations occurred more than 30 days after a prior discharge and met the inclusion criteria.

The University of Nebraska Medical Center Institutional Review Board approved this study.

\section{Data Elements}

The outcome of interest was a binary variable of unplanned 30-day readmission. Planned readmission was identified using the CMS-MUSR planned readmission algorithm which classified a list of diagnoses and procedures that were always or frequently planned and generally not considered to represent poor-quality care during $\mathrm{IH}^{22}$

The primary independent variables were utilization of 7 types of post-discharge health services: institutional outpatient, officebased primary care physician (PCP) (i.e., general practice, family practice, internal medicine, obstetrics/gynecology, geriatrics), office-based specialist, office-based non-physician practitioner (i.e., nurse practitioner, certified nurse midwife, physician assistant, physical therapist, rehabilitation, other), ED, home health care, and skilled nursing facility. Given that 30day readmission was defined as occurring any time between 0 and 30 days of discharge, we defined each post-discharge service as utilization occurring between $\mathrm{IH}$ discharge and the unplanned 30-day readmission for patients who were readmitted or within 30 days of $\mathrm{IH}$ discharge for patients who were not readmitted. Services utilized on the readmission date (or 1 day prior to readmission for ED visits) were excluded.

For each post-discharge service, we studied utilization in two ways. First, we measured whether the patient utilized each service or not, a binary variable of any visit versus no visit. Second, we used the first three digits of International Classification of Diseases, 9th revision (ICD-9) codes to determine whether the visit occurred for the same diagnosis or a different diagnosis than the IH. Thus, post-discharge services were categorized as no visit, visit for the same diagnosis as $\mathrm{IH}$, and visit for a different diagnosis than IH. We further assessed care for the same diagnosis in two separate analyses given that CMS collects up to 25 diagnosis codes per hospitalization: (a) the primary diagnosis of IH was the same as any diagnosis for post-discharge care and (b) any diagnosis of IH was the same as any diagnosis for post-discharge care.

Other explanatory variables included pre-IH care of the 7 service types listed above as indicators of prior engagement in care and characteristics of IH (i.e., weekend admission, surgical intervention, length of stay [LOS], and discharge status). A binary variable of surgical intervention during $\mathrm{IH}$ was constructed using any of up to 25 ICD-9 surgical procedure codes as defined by CMS (e.g., total hip replacement, thoracentesis, noninvasive mechanical ventilation). We also controlled for age categories, gender, race/ethnicity, the 20 most frequent clinical conditions by ICD-9 codes, the Charlson comorbidity index, health insurance status (dual eligibility for Medicare and Medicaid, participation in Medicare Part D), and geographic region.

\section{Statistical Analysis}

We employed multivariable logistic regression to quantify the association between post-discharge health services utilization and unplanned 30-day readmission after controlling for the explanatory variables listed above. ${ }^{24}$ We conducted our 
analysis for the complete study sample (all-cause $\mathrm{IH}$ ) and separately for the three clinical conditions initially targeted by the HRRP (heart failure, acute myocardial infarction [AMI], and pneumonia) to characterize post-discharge services utilization and its impact on readmission. All estimates were adjusted with a robust option and clustered by county.

\section{RESULTS}

The study sample included 583,199 patients with all-cause IH. Within this sample, 68,508 (11.7\%) had unplanned readmissions within 30 days, and only $18.1 \%$ of these readmissions shared the same primary diagnosis as the IH. For patients with IH for heart failure, AMI, and pneumonia, $17.6 \%, 13.0 \%$, and $12.4 \%$ experienced unplanned 30-day readmission, respectively (Table 1 ).

Among patients with all-cause $\mathrm{IH}$, the mean age was 78.2 years, $57.8 \%$ were female, and $83.2 \%$ were nonHispanic white. Fifty-eight percent $(58.4 \%)$ of IHs were attributable to the 20 most frequent primary diagnoses. For postdischarge services utilization, $43.7 \%, 69.8 \%$, and $71.4 \%$ respectively had any institutional outpatient, PCP, and specialist visits between discharge from IH and unplanned 30-day readmission or within 30 days of IH discharge. During the same time period, $11.1 \%$ of patients visited the ED, while $46.5 \%$, $23.9 \%$, and $23.5 \%$ respectively used non-physician practitioners, home health care, and skilled nursing facilities. When compared with the 30 days post-IH, in the 30 days prior to $\mathrm{IH}$, a higher proportion of patients visited the ED, whereas a lower proportion utilized PCPs, home health care, and skilled nursing facilities. Patients were hospitalized for an average of 4.4 days, $58.1 \%$ of patients received surgical interventions, and half of patients were discharged home to self-care. Table 2 depicts sociodemographic characteristics, post-discharge services utilization, pre-IH care, IH characteristics, primary diagnoses, and geographic region for all-cause IH and the three disease-specific cohorts.

Utilization of the 7 post-discharge services for the same or different diagnoses than the IH is presented in Figure 1 for allcause IH. In the first analysis, where we matched primary diagnosis of IH to any of up to 25 diagnoses for postdischarge services, $12.7 \%, 33.8 \%$, and $26.9 \%$ of institutional outpatient, PCP, and specialist visits shared the same primary diagnosis as IH. However, none of the post-discharge diagnoses matched the primary diagnosis of IH among $31.0 \%, 35.8 \%$, and $44.5 \%$ of institutional outpatient, PCP, and specialist visits. In the second analysis, where we matched any IH diagnosis to any diagnosis of post-discharge services, the proportion of visits for a diagnosis matching the $\mathrm{IH}$ substantially increased. However, $9.7 \%, 5.6 \%$, and $17.4 \%$ of institutional outpatient, PCP, and specialist visits occurred for completely different diagnoses than the IH. Non-physician practitioner visits followed a similar pattern. These trends in post-discharge services utilization were similar in the three disease-specific cohorts.

In multivariable logistic regression for all-cause $\mathrm{IH}$, any institutional outpatient visit was most strongly negatively associated with unplanned 30-day readmission (odds ratio [OR], $0.35 ; 95 \%$ confidence interval [CI], 0.34-0.36) followed by home health care (OR, 0.62; 95\% CI, 0.61-0.64), PCP (OR, $0.69 ; 95 \% \mathrm{CI}, 0.68-0.71)$, and specialist visits (OR, $0.75 ; 95 \%$ CI, 0.73-0.77). Non-physician practitioner visits (OR, 0.80 ; 95\% CI, 0.78-0.82) also had a negative association with readmission, while skilled nursing facilities had no statistically significant association.

ED visits were strongly positively associated with readmission (OR, 4.75; 95\% CI, 4.59-4.92). Readmission was also positively associated with non-ED pre-IH care, hospital LOS, Charlson comorbidity index, dual eligibility for Medicare and Medicaid, and participation in Medicare Part D. There was a statistically significant association between readmission and a majority of the 20 most frequent diagnoses, but the direction of association varied among these diagnoses.

For the three disease-specific cohorts, institutional outpatient visits were most strongly negatively associated with readmission, followed by specialist visits for heart failure (OR, $0.62 ; 95 \%$ CI, 0.57-0.67) and PCP visits for pneumonia (OR, 0.58; 95\% CI, 0.52-0.64). For AMI, PCP (OR, 0.65; $95 \%$ CI, 0.58-0.72), specialist, and non-physician practitioner visits had similar magnitudes of negative association with readmission. Table 3 details the results of these multivariable logistic regressions.

Table 4 shows the results of the multivariable logistic regressions exploring whether post-discharge services were utilized for the same or a different diagnosis than IH. In the first analysis, comparing the primary diagnosis for $\mathrm{IH}$ with up to 25 diagnoses for post-discharge services, institutional outpatient visits for the same diagnosis (OR, 0.33 ; 95\% CI, 0.31-0.34) and different diagnoses (OR, 0.36; 95\% CI, 0.35-0.37) were similarly negatively associated with readmission compared with no visit. In the second analysis, comparing any diagnosis for IH with any diagnosis for post-discharge services, institutional outpatient visits for the same diagnosis (OR, 0.34; $95 \%$ CI, 0.33-0.35) had a stronger negative association with

Table 1 Unplanned 30-Day Hospital Readmission: Index Hospitalization for All-Cause, Heart Failure, Acute Myocardial Infarction (AMI), and Pneumonia (No. (\%))

\begin{tabular}{lllll}
\hline \hline & All-cause $(\boldsymbol{n}=\mathbf{5 8 3 , 1 9 9 )}$ & Heart failure $(\boldsymbol{n}=\mathbf{3 0 , 4 7 3 )}$ & AMI $(\boldsymbol{n}=\mathbf{1 6 , 4 1 8})$ & Pneumonia $(\boldsymbol{n}=\mathbf{2 3 , 0 4 6})$ \\
\hline Unplanned 30-day readmission & $68,508(11.7)$ & $5357(17.6)$ & $2134(13.0)$ & $2862(12.4)$ \\
\hline
\end{tabular}


Table 2 Patient Sociodemographics, Post-Discharge Services Utilization Measured by Any Visit, Pre-Index Hospitalization (IH) Care, Characteristics of IH, and Primary Diagnoses: IH for All-Cause, Heart Failure, Acute Myocardial Infarction (AMI), and Pneumonia (No. (\%))

\begin{tabular}{|c|c|c|c|c|}
\hline & $\begin{array}{l}\text { All-cause } \\
(n=583,199)\end{array}$ & $\begin{array}{l}\text { Heart failure } \\
(n=30,473)\end{array}$ & $\begin{array}{l}\text { AMI } \\
(n=16,418)\end{array}$ & $\begin{array}{l}\text { Pneumonia } \\
(n=23,046)\end{array}$ \\
\hline \multicolumn{5}{|l|}{ Age category } \\
\hline $65-70$ & $132,545(22.7)$ & $4351(14.3)$ & $4160(25.3)$ & $3783(16.4)$ \\
\hline $71-75$ & $113,286(19.4)$ & 4623 (15.2) & 3347 (20.4) & $3808(16.5)$ \\
\hline $76-80$ & $106,854(18.3)$ & $5195(17.0)$ & 2895 (17.6) & $4153(18.0)$ \\
\hline $81-85$ & $99,183(17.0)$ & 6014 (19.8) & $2669(16.3)$ & 4345 (18.9) \\
\hline $86-90$ & $80,294(13.8)$ & 5924 (19.4) & 1993 (12.1) & $3947(17.1)$ \\
\hline 91 or above & $51,037(8.8)$ & $4366(14.3)$ & $1354(8.3)$ & $3010(13.1)$ \\
\hline Women & $336,832(57.8)$ & $16,459(54.0)$ & $7490(45.6)$ & $12,630(54.8)$ \\
\hline \multicolumn{5}{|l|}{ Race/ethnicity } \\
\hline Non-Hispanic white & $485,045(83.2)$ & $24,300(79.7)$ & $13,836(84.3)$ & $19,673(85.4)$ \\
\hline African American & $50,140(8.6)$ & 3865 (12.7) & $1185(7.2)$ & $1505(6.5)$ \\
\hline Hispanic & $29,208(5.0)$ & $1477(4.8)$ & $799(4.9)$ & $1145(5.0)$ \\
\hline Other & $18,806(3.2)$ & $831(2.7)$ & $598(3.6)$ & $723(3.1)$ \\
\hline \multicolumn{5}{|l|}{ Post-discharge health services utilization } \\
\hline Institutional outpatient & $254,970(43.7)$ & $15,137(49.7)$ & $7910(48.2)$ & $10,483(45.5)$ \\
\hline Office-based primary care physician & $406,835(69.8)$ & $23,158(76.0)$ & $10,792(65.7)$ & $18,224(79.1)$ \\
\hline Office-based specialist & $416,411(71.4)$ & $23,830(78.2)$ & $13,147(80.1)$ & $16,317(70.8)$ \\
\hline Office-based non-physician practitioner & $270,904(46.5)$ & $15,338(50.3)$ & $6796(41.4)$ & $10,288(44.6)$ \\
\hline Emergency department & $64,997(11.1)$ & 3918 (12.9) & 2069 (12.6) & $2545(11.0)$ \\
\hline Home health care & $139,428(23.9)$ & $8626(28.3)$ & $3154(19.2)$ & 5447 (23.6) \\
\hline Skilled nursing facility & $136,812(23.5)$ & $5887(19.3)$ & $2382(14.5)$ & $5160(22.4)$ \\
\hline \multicolumn{5}{|l|}{ Health services in 30 days prior to $\mathrm{IH}$} \\
\hline Institutional outpatient & $274,104(47.0)$ & $14,114(46.3)$ & $5577(34.0)$ & 9618 (41.7) \\
\hline Office-based primary care physician & $306,110(52.5)$ & $16,738(54.9)$ & $7046(42.9)$ & $11,932(51.8)$ \\
\hline Office-based specialist & $413,136(70.8)$ & $21,396(70.2)$ & $9687(59.0)$ & $15,285(66.3)$ \\
\hline Office-based non-physician practitioner & $289,266(49.6)$ & $15,285(50.2)$ & $6680(40.7)$ & $11,146(48.4)$ \\
\hline Emergency department & $135,837(23.3)$ & 7209 (23.7) & $4099(25.0)$ & $5310(23.0)$ \\
\hline Home health care & $33,831(5.8)$ & $2759(9.1)$ & $491(3.0)$ & $1451(6.3)$ \\
\hline Skilled nursing facility & $5767(1.0)$ & $408(1.3)$ & $87(0.5)$ & $299(1.3)$ \\
\hline IH weekend admission & $142,047(24.4)$ & $7845(25.7)$ & $4580(27.9)$ & $6310(27.4)$ \\
\hline Surgical intervention during $\mathrm{IH}$ & $338,613(58.1)$ & $11,125(36.5)$ & $13,630(83.0)$ & $6380(27.7)$ \\
\hline \multicolumn{5}{|l|}{ Length of stay category in IH } \\
\hline 1 day & $75,890(13.0)$ & $2352(7.7)$ & 1738 (10.6) & $1311(5.7)$ \\
\hline 2 days & $111,008(19.0)$ & $5322(17.5)$ & $3914(23.8)$ & $3655(15.9)$ \\
\hline 3 days & $128,902(22.1)$ & $6129(20.1)$ & 3193 (19.4) & $4903(21.3)$ \\
\hline 4-5 days & $131,637(22.6)$ & $8200(26.9)$ & $3210(19.6)$ & $6587(28.6)$ \\
\hline $6-10$ days & $102,066(17.5)$ & $6667(21.9)$ & $3033(18.5)$ & $5280(22.9)$ \\
\hline 11 days or longer & $33,696(5.8)$ & $1803(5.9)$ & $1330(8.1)$ & $1310(5.7)$ \\
\hline \multicolumn{5}{|l|}{ Discharge status in IH } \\
\hline Home, self-care & $294,818(50.6)$ & $15,078(49.5)$ & $10,746(65.5)$ & $12,145(52.7)$ \\
\hline Home, with care & $113,334(19.4)$ & $8229(27.0)$ & $2598(15.8)$ & $4748(20.6)$ \\
\hline Skilled nursing facility & $136,696(23.4)$ & $5832(19.1)$ & $2309(14.1)$ & $5250(22.8)$ \\
\hline Outpatient rehabilitation & $33,616(5.8)$ & $851(2.8)$ & $609(3.7)$ & $630(2.7)$ \\
\hline \multirow{2}{*}{\multicolumn{5}{|c|}{$\begin{array}{l}\text { Hospice } \\
\text { Twenty most frequent diagnoses in } \mathrm{IH}^{(\mathrm{i})}\end{array}$}} \\
\hline & & & & \\
\hline All other diagnoses (reference) & 242,868 (41.6) & $0(0.0)$ & $0(0.0)$ & $0(0.0)$ \\
\hline Osteoarthritis and allied disorders & $44,328(7.6)$ & $0(0.0)$ & $0(0.0)$ & $0(0.0)$ \\
\hline Septicemia & $33,343(5.7)$ & $0(0.0)$ & $0(0.0)$ & $0(0.0)$ \\
\hline Heart failure & $30,473(5.2)$ & $30,473(100)$ & $0(0.0)$ & $0(0.0)$ \\
\hline Cardiac dysrhythmias & $25,551(4.4)$ & $0(0.0)$ & $0(0.0)$ & $0(0.0)$ \\
\hline Pneumonia & $23,046(4.0)$ & $0(0.0)$ & $0(0.0)$ & $23,046(100)$ \\
\hline Chronic bronchitis & $17,185(2.9)$ & $0(0.0)$ & $0(0.0)$ & $0(0.0)$ \\
\hline Other disorders of urethra and urinary tract & $16,645(2.9)$ & $0(0.0)$ & $0(0.0)$ & $0(0.0)$ \\
\hline Acute myocardial infarction & $16,418(2.8)$ & $0(0.0)$ & $16,418(100)$ & $0(0.0)$ \\
\hline Fracture of neck of femur & $15,764(2.7)$ & $0(0.0)$ & $0(0.0)$ & $0(0.0)$ \\
\hline Acute renal failure & $15,156(2.6)$ & $0(0.0)$ & $0(0.0)$ & $0(0.0)$ \\
\hline Charlson comorbidity index (mean (SD)) & $1.9(1.9)$ & $3.3(1.8)$ & $2.9(1.8)$ & $2.2(1.9)$ \\
\hline Dual eligibility for Medicare and Medicaid & $104,851(18.0)$ & $6348(20.8)$ & $2445(14.9)$ & $4959(21.5)$ \\
\hline Participation in Medicare Part D & $396,019(67.9)$ & $21,067(69.1)$ & $10,418(63.5)$ & $16,002(69.4)$ \\
\hline \multicolumn{5}{|l|}{ Region } \\
\hline Northeast & $118,898(20.4)$ & $6720(22.1)$ & $3171(19.3)$ & 4458 (19.3) \\
\hline Midwest & $135,885(23.3)$ & $7417(24.3)$ & $4157(25.3)$ & $5429(23.6)$ \\
\hline South & $238,988(41.0)$ & $12,519(41.1)$ & $6578(40.1)$ & 9929 (43.1) \\
\hline West & $89,428(15.3)$ & $3817(12.5)$ & $2512(15.3)$ & $3230(14.0)$ \\
\hline
\end{tabular}

${ }^{(i)}$ Eleventh to twentieth most frequent diagnoses included occlusion of cerebral arteries (2.6\%), complications of certain specified procedures (2.2\%), other forms of chronic ischemic heart disease (2.1\%), other cellulitis and abscess (1.8\%), general symptoms (1.7\%), intestinal obstruction without mention of hernia (1.6\%), diverticula of intestine (1.6\%), disorders of electrolyte and acid-base balance (1.5\%), other diseases of lung (1.3\%), and symptoms of respiratory system and other chest (1.2\%) 
All-Cause Index Hospitalization $(n=583,199)$

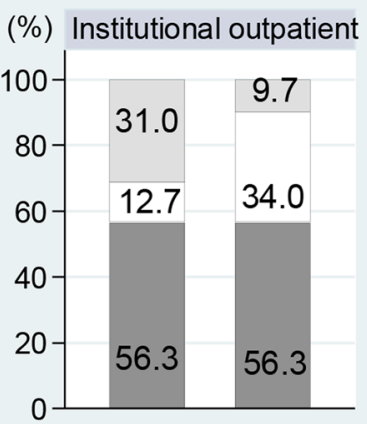

(a)

(b)

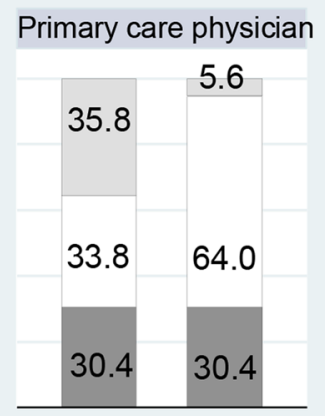

(a)

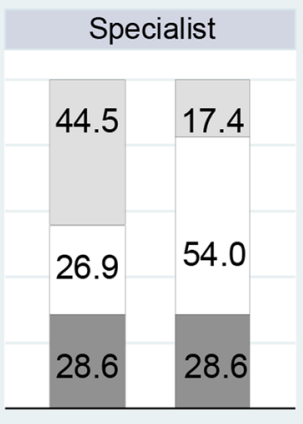

(a) (b)
Non-physician

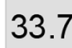

13.0

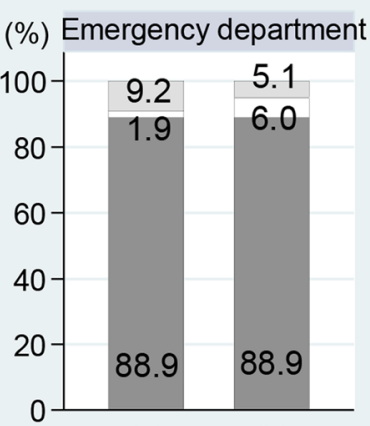

(a) (b)

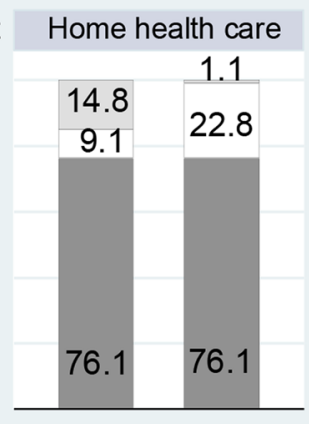

(a) (b)

Skilled nursing facility

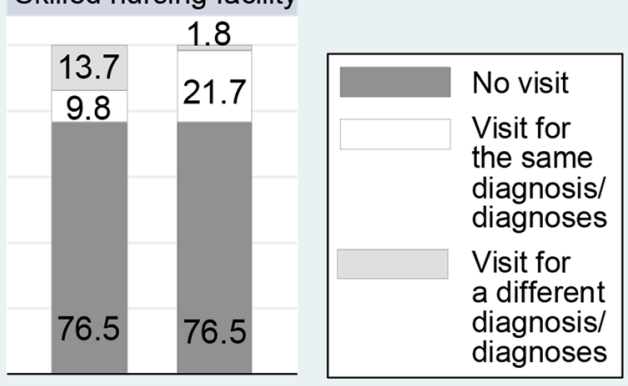

(a)

Figure 1 Post-discharge services utilization by the relatedness of diagnoses for index hospitalization and post-discharge care: all-cause index hospitalization $(n=\mathbf{5 8 3}, 199)$.

Note: (a) Primary diagnosis of index hospitalization corresponded to any diagnosis of post-discharge care. (b) Any diagnosis of index hospitalization corresponded to any diagnosis of post-discharge care.

readmission than visits for different diagnoses (OR, 0.40; 95\% CI, 0.39-0.42). PCP, specialist, and non-physician practitioner visits showed patterns similar to institutional outpatient visits.

For the three disease-specific cohorts, the association of post-discharge services utilization with unplanned 30-day readmission showed similar patterns to those for all-cause IH in most cases. However, compared with no visit, PCP visits for different diagnoses were more strongly negatively associated with readmission than visits for the same diagnosis for AMI (in assessment (a)) and pneumonia (in assessment (b)).

\section{DISCUSSION}

We examined the association between post-discharge health services utilization and unplanned 30-day readmissions for allcause IH and three health conditions included in CMS's HRRP. We found that a substantial proportion of patients utilized post-discharge services for health conditions differing from the primary diagnosis for IH. Furthermore, we provided the first empirical evidence that post-discharge services for diagnoses different than for $\mathrm{IH}$ were similarly or even more strongly associated with preventing unplanned 30 -day readmission than post-discharge services for the same diagnosis as IH.

Post-discharge care is often disproportionately focused on the primary diagnosis of $\mathrm{IH}$, which may represent a missed opportunity to improve overall patient health and to prevent readmission. ${ }^{15,}{ }^{16}$ Previous research suggests that recently discharged patients frequently develop new health problems or experience a post-hospitalization syndrome characterized by deconditioning and residual symptoms, which may lead to readmission. ${ }^{6,15,16,18-20,25}$ Furthermore, difficulties with independent daily living represent a major contributing factor to readmission. ${ }^{26}$ Therefore, improvement in general health status, as well as treatment of the acute illness that caused $\mathrm{IH}$, should both be targets of post-discharge care. ${ }^{27}$ The strong negative association reported in our study between postdischarge services utilization for numerous diagnoses and readmission substantiates this recommendation.

We used administrative claims data to characterize the full breadth of health services utilization per episode of $\mathrm{IH}$ including pre-IH care, characteristics of $\mathrm{IH}$, and post-discharge care. To our knowledge, this is the first study to examine 7 different types of health services for pre-IH and post-discharge care. Most prior intervention studies have lacked information regarding pre-IH care, had incomplete records of patients' post-discharge care due to an inability to track utilization outside of $\mathrm{IH}$ site, and focused on single type of post-discharge care. ${ }^{1,3-6,28-32}$

This study makes two novel contributions to the literature. First, we measured post-discharge services utilization occurring for the same or a different diagnosis than IH. Previous 
Table 3 Association of Post-Discharge Services Utilization with Unplanned 30-Day Readmission: Index Hospitalization (IH) for All-Cause, Heart Failure, Acute Myocardial Infarction (AMI), and Pneumonia

\begin{tabular}{|c|c|c|c|c|}
\hline & \multirow{2}{*}{$\frac{\text { All-cause }(n=583,199)}{\text { OR }(95 \% \text { CI })}$} & \multirow{2}{*}{$\frac{\text { Heart failure }(n=30,473)}{\text { OR }(95 \% \text { CI })}$} & \multirow{2}{*}{$\frac{\mathrm{AMI}(n=16,418)}{\mathrm{OR}(95 \% \mathrm{CI})}$} & \multirow{2}{*}{$\frac{\text { Pneumonia }(n=23,046)}{\text { OR }(95 \% \text { CI })}$} \\
\hline & & & & \\
\hline \multicolumn{5}{|l|}{ Post-discharge health services utilization } \\
\hline Institutional outpatient & $0.35(0.34-0.36)$ & $0.36(0.33-0.38)$ & $0.26(0.22-0.29)$ & $0.34(0.30-0.37)$ \\
\hline Office-based primary care physician & $0.69(0.68-0.71)$ & $0.68(0.63-0.73)$ & $0.65(0.58-0.72)$ & $0.58(0.52-0.64)$ \\
\hline Office-based specialist & $0.75(0.73-0.77)$ & $0.62(0.57-0.67)$ & $0.67(0.59-0.75)$ & $0.71(0.64-0.78)$ \\
\hline Office-based non-physician practitioner & $0.80(0.78-0.82)$ & $0.71(0.66-0.76)$ & $0.65(0.58-0.72)$ & $0.77(0.70-0.84)$ \\
\hline Emergency department & $4.75(4.59-4.92)$ & $4.45(4.08-4.86)$ & $5.27(4.57-6.09)$ & $4.47(3.95-5.07)$ \\
\hline Home health care & $0.62(0.61-0.64)$ & $0.75(0.69-0.81)$ & $0.71(0.61-0.83)$ & $0.68(0.59-0.77)$ \\
\hline Skilled nursing facility & $0.98(0.94-1.02)$ & $0.77(0.64-0.91)$ & $1.17(0.85-1.61)$ & $0.74(0.61-0.90)$ \\
\hline \multicolumn{5}{|l|}{ Health services in 30 days prior to $\mathrm{IH}$} \\
\hline Institutional outpatient & $1.33(1.30-1.36)$ & $1.31(1.23-1.41)$ & $1.35(1.20-1.52)$ & $1.44(1.31-1.59)$ \\
\hline Office-based primary care physician & $1.15(1.12-1.17)$ & $1.21(1.12-1.30)$ & $1.18(1.06-1.31)$ & $1.15(1.05-1.25)$ \\
\hline Office-based specialist & $1.19(1.16-1.21)$ & $1.13(1.05-1.23)$ & $1.17(1.03-1.32)$ & $1.25(1.13-1.39)$ \\
\hline Office-based non-physician practitioner & $1.12(1.10-1.14)$ & $1.14(1.06-1.22)$ & $1.20(1.08-1.35)$ & $1.17(1.07-1.29)$ \\
\hline Emergency department & $1.01(0.99-1.03)$ & $1.04(0.97-1.12)$ & $0.91(0.80-1.03)$ & $0.92(0.82-1.02)$ \\
\hline Home health care & $1.12(1.08-1.16)$ & $1.17(1.05-1.31)$ & $1.16(0.91-1.47)$ & $1.22(1.06-1.42)$ \\
\hline Skilled nursing facility & $1.47(1.37-1.58)$ & $1.21(0.93-1.56)$ & $1.09(0.63-1.90)$ & $1.47(1.07-2.02)$ \\
\hline IH weekend admission & $1.00(0.98-1.02)$ & $0.97(0.90-1.04)$ & $0.93(0.83-1.03)$ & $0.99(0.90-1.09)$ \\
\hline Surgical intervention during IH & $1.01(0.99-1.03)$ & $1.01(0.94-1.08)$ & $0.96(0.83-1.11)$ & $1.31(1.19-1.43)$ \\
\hline \multicolumn{5}{|l|}{ Length of stay category in IH } \\
\hline 1 day & 1.00 (reference) & 1.00 (reference) & 1.00 (reference) & 1.00 (reference) \\
\hline 2 days & $1.16(1.12-1.19)$ & $1.19(1.03-1.38)$ & $1.28(1.04-1.58)$ & $1.03(0.83-1.29)$ \\
\hline 3 days & $1.26(1.22-1.30)$ & $1.22(1.06-1.40)$ & $1.26(1.01-1.57)$ & $1.12(0.91-1.38)$ \\
\hline 4-5 days & $1.48(1.43-1.53)$ & $1.38(1.20-1.58)$ & $1.73(1.40-2.14)$ & $1.28(1.05-1.57)$ \\
\hline $6-10$ days & $1.79(1.73-1.85)$ & $1.64(1.42-1.90)$ & $1.95(1.56-2.44)$ & $1.41(1.15-1.73)$ \\
\hline 11 days or longer & $2.42(2.31-2.53)$ & $2.06(1.72-2.48)$ & $2.38(1.82-3.11)$ & $1.78(1.40-2.27)$ \\
\hline \multicolumn{5}{|l|}{ Discharge status in IH } \\
\hline Home, self-care & 1.00 (reference) & 1.00 (reference) & 1.00 (reference) & 1.00 (reference) \\
\hline Home, with care & $1.52(1.48-1.56)$ & $1.35(1.24-1.48)$ & $1.64(1.38-1.95)$ & $1.50(1.31-1.71)$ \\
\hline Skilled nursing facility & $1.10(1.06-1.15)$ & $1.29(1.08-1.54)$ & $1.11(0.80-1.53)$ & $1.54(1.26-1.89)$ \\
\hline Outpatient rehabilitation & $0.44(0.40-0.48)$ & $0.44(0.33-0.58)$ & $0.42(0.30-0.58)$ & $0.61(0.43-0.85)$ \\
\hline Hospice & $0.19(0.16-0.22)$ & $0.12(0.08-0.19)$ & $0.07(0.02-0.21)$ & $0.08(0.03-0.18)$ \\
\hline \multicolumn{5}{|l|}{ Twenty most frequent diagnoses in $\mathrm{IH}$} \\
\hline All other diagnoses (reference) & 1.00 (reference) & - & - & - \\
\hline Osteoarthritis and allied disorders & $0.42(0.39-0.44)$ & - & - & - \\
\hline Septicemia & $1.18(1.13-1.22)$ & - & - & - \\
\hline Heart failure & $1.43(1.38-1.48)$ & - & - & - \\
\hline Cardiac dysrhythmias & $1.24(1.18-1.29)$ & - & - & - \\
\hline Pneumonia & $1.04(1.00-1.09)$ & - & - & - \\
\hline Chronic bronchitis & $1.30(1.24-1.36)$ & - & - & - \\
\hline Other disorders of urethra/urinary tract & $1.18(1.12-1.24)$ & - & - & - \\
\hline Acute myocardial infarction & $1.11(1.06-1.17)$ & - & - & - \\
\hline Fracture of neck of femur & $0.84(0.79-0.89)$ & - & - & - \\
\hline Acute renal failure & $0.80(0.75-0.84)$ & - & - & - \\
\hline Occlusion of cerebral arteries & $1.29(1.23-1.35)$ & - & - & - \\
\hline Complications of specified procedures & $1.14(1.08-1.21)$ & - & - & - \\
\hline Other chronic ischemic heart disease & $1.00(0.95-1.06)$ & - & - & - \\
\hline Other cellulitis and abscess & $0.94(0.88-1.01)$ & - & - & - \\
\hline General symptoms & $0.87(0.81-0.94)$ & - & - & - \\
\hline Intestinal obstruction without hernia & $1.07(1.01-1.14)$ & - & - & - \\
\hline Diverticula of intestine & $1.05(0.98-1.12)$ & - & - & - \\
\hline Disorders of electrolyte & $1.29(1.21-1.38)$ & - & - & - \\
\hline Other diseases of lung & $1.34(1.25-1.42)$ & - & - & - \\
\hline Symptoms of respiratory system & $0.89(0.82-0.97)$ & - & - & - \\
\hline Charlson comorbidity index & $1.13(1.13-1.14)$ & $1.08(1.06-1.10)$ & $1.18(1.15-1.22)$ & $1.11(1.09-1.14)$ \\
\hline Dual eligibility for Medicare and Medicaid & $1.14(1.12-1.17)$ & $1.20(1.10-1.30)$ & $0.93(0.81-1.08)$ & $1.08(0.97-1.21)$ \\
\hline Participation in Medicare Part D & $1.10(1.08-1.13)$ & $1.08(1.00-1.16)$ & $1.22(1.09-1.37)$ & $1.09(0.99-1.20)$ \\
\hline
\end{tabular}

In multivariable logistic regressions, odds ratio (OR) and 95\% confidence interval (CI) were adjusted for age categories, gender, racelethnicity, and geographic region categories as well as for the variables listed in the table

studies suggested that multidisciplinary post-discharge interventions (e.g., patient discharge instruction by nurses in the hospital and follow-up health education in home health care) were more effective than single-dimensional care due to the diverse causes of readmission. ${ }^{5,33-36}$ However, no previous study has examined whether the post-discharge interventions were targeted at the same or different diagnoses than $\mathrm{IH}$. We used ICD-9 codes to differentiate post-discharge services for the same and different diagnoses than IH and to examine systematically how services for the same or different diagnoses than $\mathrm{IH}$ were associated with readmission.

The HRRP definition of diagnosis may be unbalanced because the Program targets selected primary diagnoses for IH (e.g., heart failure, AMI, pneumonia), while the penalty is applied for all-cause for readmission. As such, our first assessment of care for the same diagnosis as IH (i.e., primary 
Table 4 Association of Post-Discharge Services Utilization for the Same or Different Diagnosis Than Index Hospitalization with Unplanned 30Day Readmission: All-Cause, Heart Failure, Acute Myocardial Infarction (AMI), and Pneumonia

\begin{tabular}{|c|c|c|c|c|c|c|c|c|}
\hline & \multicolumn{2}{|c|}{$\begin{array}{l}\text { All-cause } \\
(n=583,199)\end{array}$} & \multicolumn{2}{|c|}{$\begin{array}{l}\text { Heart failure } \\
(n=30,473)\end{array}$} & \multicolumn{2}{|c|}{$\begin{array}{l}\text { AMI } \\
(n=16,418)\end{array}$} & \multicolumn{2}{|c|}{$\begin{array}{l}\text { Pneumonia } \\
(n=23,046)\end{array}$} \\
\hline & \multirow{2}{*}{$\frac{\text { (a) }}{\text { OR }}$} & \multirow{2}{*}{$\frac{\text { (b) }}{\text { OR }}$} & \multirow{2}{*}{$\frac{\text { (a) }}{\text { OR }}$} & \multirow{2}{*}{$\frac{\text { (b) }}{\text { OR }}$} & \multirow{2}{*}{$\frac{\text { (a) }}{\text { OR }}$} & \multirow{2}{*}{$\frac{\text { (b) }}{\text { OR }}$} & \multirow{2}{*}{$\frac{\text { (a) }}{\text { OR }}$} & \multirow{2}{*}{$\frac{\text { (b) }}{\text { OR }}$} \\
\hline & & & & & & & & \\
\hline \multicolumn{9}{|l|}{ Institutional outpatient } \\
\hline No visit & $1.00-$ & $1.00-$ & $1.00-$ & $1.00-$ & $1.00-$ & $1.00-$ & $1.00-$ & $1.00-$ \\
\hline Visit for the same diagnosis & $0.33^{*}$ & $0.34 *$ & $0.33^{*}$ & $0.35^{*}$ & $0.19 *$ & $0.24 *$ & $0.31 *$ & $0.33 *$ \\
\hline Visit for a different diagnosis & $0.36^{*}$ & $0.40^{*}$ & $0.40 *$ & $0.43 *$ & $0.28^{*}$ & $0.34 *$ & $0.34 *$ & $0.39 *$ \\
\hline \multicolumn{9}{|c|}{ Office-based primary care physician } \\
\hline No visit & $1.00-$ & $1.00-$ & $1.00-$ & $1.00-$ & $1.00-$ & $1.00-$ & $1.00-$ & $1.00-$ \\
\hline Visit for the same diagnosis & $0.69^{*}$ & $0.69^{*}$ & $0.67 *$ & $0.67 *$ & $0.72 *$ & $0.65^{*}$ & $0.58^{*}$ & $0.58 *$ \\
\hline Visit for a different diagnosis & $0.69^{*}$ & $0.74 *$ & $0.68^{*}$ & $0.69^{*}$ & $0.60 *$ & $0.73^{*}$ & $0.57 *$ & $0.52 *$ \\
\hline \multicolumn{9}{|l|}{ Office-based specialist } \\
\hline No visit & $1.00-$ & $1.00-$ & $1.00-$ & $1.00-$ & $1.00-$ & $1.00-$ & $1.00-$ & $1.00-$ \\
\hline Visit for the same diagnosis & $0.71 *$ & $0.73^{*}$ & $0.58 *$ & $0.59 *$ & $0.69^{*}$ & $0.62 *$ & $0.72 *$ & $0.68 *$ \\
\hline Visit for a different diagnosis & $0.77 *$ & $0.82 *$ & $0.65^{*}$ & $0.77 *$ & $0.65^{*}$ & 1.03 & $0.71 *$ & $0.78 *$ \\
\hline \multicolumn{9}{|c|}{ Office-based non-physician practitioner } \\
\hline No visit & $1.00-$ & $1.00-$ & $1.00-$ & $1.00-$ & $1.00-$ & $1.00-$ & $1.00-$ & $1.00-$ \\
\hline Visit for the same diagnosis & $0.75^{*}$ & $0.74 *$ & $0.67 *$ & $0.66^{*}$ & $0.59 *$ & $0.57 *$ & $0.67 *$ & $0.69 *$ \\
\hline Visit for a different diagnosis & $0.82^{*}$ & $0.93^{*}$ & $0.74 *$ & $0.89^{\dagger}$ & $0.67 *$ & $0.87^{\dagger}$ & $0.79 *$ & 0.94 \\
\hline \multicolumn{9}{|l|}{ Emergency department } \\
\hline No visit & $1.00-$ & $1.00-$ & $1.00-$ & $1.00-$ & $1.00-$ & $1.00-$ & $1.00-$ & $1.00-$ \\
\hline Visit for the same diagnosis & $5.21 *$ & $4.94 *$ & $5.59 *$ & $4.69 *$ & $6.62 *$ & $5.17 *$ & $4.62 *$ & $4.78^{*}$ \\
\hline Visit for a different diagnosis & $4.65 *$ & $4.51 *$ & $4.04 *$ & $3.77 *$ & $5.14 *$ & $4.98^{*}$ & $4.42 *$ & $3.97^{*}$ \\
\hline \multicolumn{9}{|l|}{ Home health care } \\
\hline No visit & $1.00-$ & $1.00-$ & $1.00-$ & $1.00-$ & $1.00-$ & $1.00-$ & $1.00-$ & $1.00-$ \\
\hline Visit for the same diagnosis & $0.71 *$ & $0.63 *$ & $0.76^{*}$ & $0.75^{*}$ & $0.75^{*}$ & $0.72 *$ & $0.75^{*}$ & $0.68 *$ \\
\hline Visit for a different diagnosis & $0.57 *$ & $0.46^{*}$ & $0.67 *$ & 0.44 & $0.65 *$ & 0.46 & $0.62 *$ & 0.50 \\
\hline \multicolumn{9}{|l|}{ Skilled nursing facility } \\
\hline No visit & $1.00-$ & $1.00-$ & $1.00-$ & $1.00-$ & $1.00-$ & $1.00-$ & $1.00-$ & $1.00-$ \\
\hline Visit for the same diagnosis & 0.97 & 0.98 & $0.80^{\dagger}$ & $0.77 *$ & 1.02 & 1.16 & $0.78^{\dagger}$ & $0.76^{*}$ \\
\hline Visit for a different diagnosis & 0.99 & 1.03 & $0.71 *$ & 0.67 & 1.29 & 1.41 & $0.73 *$ & $0.61^{\dagger}$ \\
\hline
\end{tabular}

Notes: (a) Primary diagnosis of index hospitalization corresponded to any diagnosis of post-discharge care. (b) Any diagnosis of index hospitalization corresponded to any diagnosis of post-discharge care

(ii) In multivariable logistic estimations, odds ratio (OR) and 95\% confidence interval (CI) were adjusted for age categories, gender, racelethnicity, 7 types of health services used in the 30 days prior to index hospitalization $(I H)$, IH weekend admission, surgical intervention during IH, hospital length of stay categories in IH, discharge status in IH, 20 most frequent diagnoses (all-cause IH only), Charlson comorbidity index, dual eligibility for Medicare and Medicaid, participation in Medicare Part D, and geographic region categories as well as for the variables listed in the table

(iii) $* P<0.01,{ }^{\dagger} P<0.05$, - reference

diagnosis of IH corresponded to any diagnosis of postdischarge service) better reflects the current application of the CMS HRRP, which was recently associated with increased patient mortality. ${ }^{37}$ However, the second assessment of care for the same diagnosis as IH (i.e., any diagnosis of IH corresponded to any diagnosis of post-discharge care) could provide a template for future readmission reduction interventions given that not only the primary diagnosis of $\mathrm{IH}$ is associated with readmission.

Second, our study population included all-cause IH and compared whether patterns of post-discharge services utilization and readmission were similar to the three disease-specific cohorts initially included in the CMS HRRP. Most studies relating readmission have focused on the three diseasespecific cohorts, which constituted only $12.0 \%$ of the sample population in our study, and provided no data regarding the generalizability of their interventions or outcomes for other health conditions. ${ }^{4-7,11,17,19,29-31,37,38}$

All-cause IH is rarely a study target because it is difficult to design preventative interventions, and very few studies analyzed all-cause IH using simple bivariate analysis. ${ }^{39,}{ }^{40}$ However, the CMS HRRP was observed to have a spillover effect: it reduced readmission for non-targeted diagnoses as well as for targeted diagnoses. ${ }^{41,} 42$ Furthermore, the Medicare Payment Advisory Commission has proposed expanding its readmission penalty program to cover all-cause $\mathrm{IH}^{43}$ based upon the broad readmission prevention benefits of post-discharge care and the fact that current efforts target only a small fraction of patients at risk of readmission. ${ }^{11,17}$ Recently, CMS applied a 30-day all-cause hospital readmission measures to medical practitioners participating in a Medicare Shared Savings Program Accountable Care Organization and included their performance in the Annual Quality and Resource Use Reports and in the Quality Composite Score for the 2017 Value Modifier. $^{44,}{ }^{45}$ These policy initiatives necessitate an expanded understanding of disease-specific and all-cause IH and the relationship of the full spectrum of post-discharge care to readmission following all-cause $\mathrm{IH}$.

Total 30-day readmission has markedly decreased in the last decade. ${ }^{2,42,46}$ In 2003, $19.6 \%$ of total hospitalizations resulted in 30-day readmission. ${ }^{1}$ However, in 2015, a study of Medicare beneficiaries showed readmission rates for HRRPtargeted and non-targeted conditions of $17.8 \%$ and $13.1 \%$ respectively. ${ }^{42}$ Furthermore, the Medicare Payment Advisory 
Commission reported that the potentially preventable readmission rate for all-cause IH had declined to $11.0 \%$ in $2014 .^{46}$ However, most readmissions are still unplanned and potentially preventable, ${ }^{30}$ and reducing hospital readmission remains a major area of health policy concern due to the potential for large-scale health care cost savings. ${ }^{1,47}$

Medicare has implemented diverse interventions such as Transitional Care Management to improve post-discharge care and thereby reduce readmission rate and costs. ${ }^{13,14}$ However, hospitals have limited time and resources to coordinate followup care, ${ }^{14,30}$ and not all intervention strategies are effective. ${ }^{4-7}$ Sometimes, patients decline post-discharge interventions due to perceived complexity and expense. ${ }^{4,48,49}$ Our study suggests that physicians should consider a holistic approach to post-discharge care because utilization of post-discharge services of various types and for various diagnoses contributes to reduced readmission. Furthermore, medical practitioners should consider patients' financial and non-financial (e.g., travel time) constraints when planning post-discharge care to optimize access to the various health services required. A wide range of health conditions causes readmissions, and general health management in outpatient settings may prevent readmissions. Indeed, some experts suggest that a model of more nurse practitioners and physician assistants under physician supervision could improve access to and timeliness of post-discharge care. $^{30}$

Our study has several important limitations. First, in an observational study, we could not control for potential unobserved confounders such as selection of specific post-discharge services. To mitigate this problem, we controlled for health status including the 20 most frequent $\mathrm{IH}$ diagnoses and the Charlson comorbidity index. Second, Medicare claims data lack information regarding patients' socioeconomic status that may influence readmission patterns. ${ }^{6,21,50}$ In this vein, we included two insurance status variables and clustered the observations by county, reasoning that the residents of each county would have similar dwelling, social, and medical infrastructure characteristics. Third, our results may not be generalizable beyond Medicare FFS beneficiaries because Medicare FFS does not require PCP referral or care within a provider network, thereby allowing flexibility in post-discharge services.

In summary, this study highlights the role of post-discharge services utilization for various diagnoses in reducing unplanned 30-day readmission. These findings may inform strategies to reduce unplanned 30-day readmissions more effectively.

Corresponding Author: Hyo Jung Tak, PhD; Department of Health Services Research and Administration University of Nebraska Medical Center, 984350 Nebraska Medical Center, Omaha, NE 68198, USA (e-mail: hyojung.tak@unmc.edu).

Contributors: Dr. Tak had full access to all of the data in the study and takes the responsibility for the integrity of the data and the accuracy of the data analysis. All authors have made substantial contributions to this manuscript and attest to the validity and legitimacy of the data as well as its interpretation. We do not have any other contributors who were not listed in the authors.

Compliance with Ethical Standards: The University of Nebraska Medical Center Institutional Review Board approved this study.

Conflict of Interest: The authors declare that they do not have a conflict of interest.

Prior Presentations: None.

\section{REFERENCES}

1. Jencks SF, Williams MV, Coleman EA. Rehospitalizations among patients in the Medicare fee-for-service program. $N$ Engl $J$ Med. 2009;360(14):1418-28.

2. Crosson F, Christianson J, Miller M. Report to the Congress: Medicare and the Health Care Delivery System. Washington, D.C:Medicare Payment Advisory Commission; 2017.

3. Jack BW, Chetty VK, Anthony D, et al. A reengineered hospital discharge program to decrease rehospitalization: a randomized trial. Ann Intern Med. 2009;150(3):178-87.

4. Leppin AL, Gionfriddo MR, Kessler M, et al. Preventing 30-day hospital readmissions: a systematic review and meta-analysis of randomized trials. JAMA Intern Med. 2014; 174(7): 1095-107.

5. Hansen LO, Young RS, Hinami K, Leung A, Williams MV. Interventions to reduce 30-day rehospitalization: a systematic review. Ann Intern Med. 2011;155(8):520-8.

6. Kansagara D, Englander $\mathbf{H}$, Salanitro A, et al. Risk prediction models for hospital readmission: a systematic review. JAMA. 2011;306(15):1688-98.

7. van Walraven C, Jennings A, Forster AJ. A meta-analysis of hospital 30-day avoidable readmission rates. J Eval Clin Pract. 2012;18(6):12118.

8. Nuckols TK, Keeler E, Morton S, et al. Economic evaluation of quality improvement interventions designed to prevent hospital readmission: a systematic review and meta-analysis. JAMA Intern Med. 2017;177(7):975-85.

9. Zimlichman E, Henderson D, Tamir O, et al. Health care-associated infections: a meta-analysis of costs and financial impact on the US health care system. JAMA Intern Med. 2013;173(22):2039-46.

10. Pannick S, Davis R, Ashrafian H, et al. Effects of interdisciplinary team care interventions on general medical wards: a systematic review. JAMA Intern Med. 2015;175(8): 1288-98.

11. Dharmarajan K, Krumholz HM. Strategies to reduce 30-day readmissions in older patients hospitalized with heart failure and acute myocardial infarction. Curr Geriatr Rep. 2014;3(4):306-15.

12. Peikes D, Chen A, Schore J, Brown R. Effects of care coordination on hospitalization, quality of care, and health care expenditures among Medicare beneficiaries: 15 randomized trials. JAMA. 2009;301(6):603-18.

13. Ballard J, Rankin W, Roper KL, Weatherford S, Cardarelli R. Effect of ambulatory transitional care management on 30-day readmission rates. Am J Med Qual. 2018 33(6):583-589

14. Bindman AB, Cox DF. Changes in health care costs and mortality associated with transitional care management services after a discharge among Medicare beneficiaries. JAMA Intern Med. 2018;178(9):1165-71.

15. van Walraven C, Bennett C, Jennings A, Austin PC, Forster AJ. Proportion of hospital readmissions deemed avoidable: a systematic review. CMAJ. 2011;183(7):E391-402.

16. Krumholz HM. Post-hospital syndrome-an acquired, transient condition of generalized risk. $N$ Engl J Med. 2013;368(2):100-2.

17. Dharmarajan $\mathbf{K}$, Hsieh AF, Lin Z, et al. Diagnoses and timing of 30-day readmissions after hospitalization for heart failure, acute myocardial infarction, or pneumonia. JAMA. 2013;309(4):355-63.

18. Hoyer EH, Needham DM, Atanelov L, Knox B, Friedman M, Brotman DJ. Association of impaired functional status at hospital discharge and subsequent rehospitalization. J Hosp Med. 2014;9(5):277-82.

19. Nguyen HQ, Chu L, Amy Liu IL, et al. Associations between physical activity and 30-day readmission risk in chronic obstructive pulmonary disease. Ann Am Thorac Soc. 2014;11(5):695-705.

20. Brownlee SA, Blackwell RH, Blanco BA, et al. Impact of post-hospital syndrome on outcomes following elective, ambulatory surgery. Ann Surg. 2017;266(2):274-9. 
21. Ashton CM, Wray NP. A conceptual framework for the study of early readmission as an indicator of quality of care. Soc Sci Med. 1996;43(11):1533-41.

22. Horwitz LI, Grady JN, Dorsey KB, et al. 2014 Measure Updates and Specification Report: Hospital-Wide All-Cause Unplanned Readmission Version 3.0. Prepared for the Centers for Medicare and Medicaid Services. New Haven, CT: Yale New Haven Health Services Corporation/Center for Outcomes Research \& Evaluation; 2014

23. Horwitz LI, Partovian C, Lin Z, et al. Hospital-Wide (All-Condition) 30Day Risk-Standardized Readmission Measure: Measure Methodology Report. Prepared for the Centers for Medicare and Medicaid Services. New Haven, CT: Yale New Haven Health Services Corporation/Center for Outcomes Research \& Evaluation; 2011.

24. Hosmer DW, Jr., Lemeshow SA, Sturdivant RX. Applied Logistic Regression. 3rd ed. Hoboken, NJ: Wiley; 2013.

25. Gill TM, Allore HG, Gahbauer EA, Murphy TE. Change in disability after hospitalization or restricted activity in older persons. JAMA. 2010;304(17):1919-28.

26. Kangovi S, Grande D, Meehan P, Mitra N, Shannon R, Long JA. Perceptions of readmitted patients on the transition from hospital to home. J Hosp Med. 2012;7(9):709-12.

27. Kortebein P. Rehabilitation for hospital-associated deconditioning. Am J Phys Med Rehabil. 2009;88(1):66-77.

28. Reed RL, Pearlman RA, Buchner DM. Risk factors for early unplanned hospital readmission in the elderly. J Gen Intern Med. 1991;6(3):223-8.

29. Chin MH, Goldman L. Factors contributing to the hospitalization of patients with congestive heart failure. Am J Public Health. 1997;87(4):643-8.

30. Hernandez AF, Greiner MA, Fonarow GC, et al. Relationship between early physician follow-up and 30-day readmission among Medicare beneficiaries hospitalized for heart failure. JAMA. 2010;303(17):1716-22.

31. Sharma G, Kuo YF, Freeman JL, Zhang DD, Goodwin JS. Outpatient follow-up visit and 30-day emergency department visit and readmission in patients hospitalized for chronic obstructive pulmonary disease. Arch Intern Med. 2010;170(18):1664-70.

32. Weinberger M, Oddone EZ, Henderson WG. Does increased access to primary care reduce hospital readmissions? Veterans affairs cooperative study group on primary care and hospital readmission. $N$ Engl $J$ Med. 1996;334(22): 1441-7.

33. Rich MW, Beckham V, Wittenberg C, Leven CL, Freedland KE, Carney RM. A multidisciplinary intervention to prevent the readmission of elderly patients with congestive heart failure. NEnglJ Med. 1995;333(18):1190-5.

34. Sochalski J, Jaarsma T, Krumholz HM, et al. What works in chronic care management: the case of heart failure. Health Aff (Millwood). 2009;28(1):179-89.

35. Davidson PL, Andersen RM, Wyn R, Brown ER. A framework for evaluating safety-net and other community-level factors on access for low-income populations. Inquiry. 2004;41(1):21-38.

36. Koehler F, Winkler S, Schieber $\mathbf{M}$, et al. Telemedical interventional monitoring in heart failure (TIM-HF), a randomized, controlled intervention trial investigating the impact of telemedicine on mortality in ambulatory patients with heart failure: study design. Eur $J$ Heart Fail. 2010;12(12):1354-62
37. Wadhera RK, Joynt Maddox KE, Wasfy JH, Haneuse S, Shen C, Yeh RW. Association of the hospital readmissions reduction program with mortality among Medicare beneficiaries hospitalized for heart failure, acute myocardial infarction, and pneumonia. JAMA. 2018;320(24):254252 .

38. Bueno H, Ross JS, Wang Y, et al. Trends in length of stay and short-term outcomes among Medicare patients hospitalized for heart failure, 19932006. JAMA. 2010;303(21):2141-7.

39. Vest JR, Gamm LD, Oxford BA, Gonzalez MI, Slawson KM. Determinants of preventable readmissions in the United States: a systematic review. Implement Sci. 2010;5:88.

40. Kaboli PJ, Go JT, Hockenberry J, et al. Associations between reduced hospital length of stay and 30-day readmission rate and mortality: 14year experience in 129 Veterans Affairs hospitals. Ann Intern Med. 2012;157(12):837-45.

41. Ibrahim AM, Nathan H, Thumma JR, Dimick JB. Impact of the hospital readmission reduction program on surgical readmissions among Medicare beneficiaries. Ann Surg. 2017;266(4):617-24.

42. Zuckerman RB, Sheingold SH, Orav EJ, Ruhter J, Epstein AM. Readmissions, observation, and the hospital readmissions reduction program. N Engl J Med. 2016;374(16): 1543-51.

43. Hackbarth G, Berenson R, Miller M. Report to the Congress: Medicare and the Health Care Delivery Sytem. Washington, D.C.: Medicare Payment Advisory Comission; 2013.

44. CMS Measure Information Form. 2015 Measure Information about the 30Day All-Cause Hospital Readmission Measure, Calculated for the ValueBased Payment Modifier Program. Baltimore, MD: Centers for Medicare and Medicaid Services; 2017.

45. CMS Detailed Methodology. Detailed Methodology for the 2017 Value Modifier and the 2015 Quality and Resource Use Report. Baltimore, MD: Centers for Medicare and Medicaid Services; 2017.

46. Crosson F, Christianson J, Miller M. Report to the Congress: Medicare Payment Policy. Washington, D.C.: Medicare Payment Advisory Commission; 2017.

47. Carey $\mathbf{K}$, Stefos $\mathbf{T}$. The cost of hospital readmissions: evidence from the VA. Health Care Manag Sci. 2016;19(3):241-8.

48. Naylor MD, Brooten D, Campbell R, et al. Comprehensive discharge planning and home follow-up of hospitalized elders: a randomized clinical trial. JAMA. 1999;281(7):613-20.

49. Koehler BE, Richter KM, Youngblood L, et al. Reduction of 30-day postdischarge hospital readmission or emergency department (ED) visit rates in high-risk elderly medical patients through delivery of a targeted care bundle. J Hosp Med. 2009;4(4):211-8.

50. Calvillo-King L, Arnold D, Eubank KJ, et al. Impact of social factors on risk of readmission or mortality in pneumonia and heart failure: systematic review. J Gen Intern Med. 2013;28(2):269-82.

Publisher's Note Springer Nature remains neutral with regard to jurisdictional claims in published maps and institutional affiliations. 